\title{
One method for robust control of uncertain systems - Theory and practice
}

\author{
George Leitmann \\ College of Engineering, University of California \\ Berkeley, CA 94720, U.S.A.
}

\begin{abstract}
We present a controller design methodology for uncertain systems which is based on the constructive use of Lyapunov stability theory. The uncertainties, which are deterministic, are characterized by certain structural conditions and known as well as unknown bounds. As a consequence of the Lyapunov approach, the methodology is not restricted to linear or time-invariant systems. The robustness of these controllers in the presence of singular perturbations is considered. The situation in which the full state of the system is not available for measurement is also considered as are other generalizations. Applications of the proposed discussed in the complete version of the paper.
\end{abstract}

\section{Keywords}

uncertain systems; deterministic control; robust control; resource management

\section{INTRODUCTION}

A fundamental feedback control problem* is that of obtaining some specified desired behavior from a system about which there is incomplete or uncertain information. Here we consider systems whose uncertainties are characterized deterministically rather that stochastically or fuzzily; for a stochastic approach see (Bass, 1957), and for fuzzy one see (Klir and Folger, 1988).

Our model of an uncertain system is of the form

$\dot{x}(t)=F(t, x(t), u(t), \omega)$

where $t \in I R$ is the "time" variable, $x(t) \in I R^{n}$ is the state and $u(t) \in I R^{m}$ is the control input. All the uncertainty in the system is represented by the lumped uncertain element $\omega \in \Omega$. It could be an element of $I R^{q}$ representing constant unknown parameters and inputs; it could also be a function from $I R$ into $I R^{q}$ representing unknown time varying

\footnotetext{
*Throughout this paper, references are intended to be representative rather than exhaustive. For a more complete bibliography see (Leitmann, 1990 and 1993).
} 
parameters and inputs; it could also be a function from $I R \times I R^{n} \times I R^{m}$ into $I R^{q}$ representing nonlinear elements which are difficult to characterize exactly; it could be merely an index. $F: I R \times I R^{n} \times I R^{m} \times \Omega \rightarrow I R^{n}$ is known. The only information assumed about $\omega$ is the knowledge of a nonempty set $\Omega$ to which it belongs. A related characterization of uncertainties is via inclusions see (Kurzhanskii, 1983).

Discrete systems are usually modelled by a difference equation

$x(k+1)=F(k, x(k), u(k))$

where $k \in \mathbb{Z}$ is the "time", $x(k) \in I R^{n}$ is the state, $u(k) \in I R^{m}$ is the control, and $F$ is not known but rather belongs to a set $F$, with $F$ known.

\section{CONTINUOUS SYSTEM CONTROL}

For continuous systems modelled by ordinary differential equations of the form (1) we consider control to be given by a memoryless state feedback controller

$u(t)=p(t, x(t))$.

Ideally we wish to choose $p: I R \times I R^{n} \rightarrow I R^{m}$ so that the feedback controlled system

$\dot{x}(t)=f(t, x(t), \omega)$,

where

$f(t, x, \omega):=F(t, x, p(t, x), \omega)$

has the property of g.u.a.s. (global uniform asymptotic stability) about the zero state for all $\omega \in \Omega$ and for all initial states in $I R^{n}$. However to assure g.u.a.s. of an uncertain system one sometimes has to resort to controllers which are discontinuous in the state; see (Gutman and Leitmann, 1976). To avoid such discontinuous controllers, we relax the problem to that of obtaining a family of controllers which assure that the behavior of (1) can be made arbitrarily close to g.u.a.s.; such a family is called a practically stabilizing family see (Corless and Leitmann, 1988, Corless et al., 1990).

\subsection{A Specific Class of Uncertain Continuous Systems}

An uncertain continuous system under consideration here is described by (1) and satisfies the following assumption.

Assumption C.1. $\dagger^{\dagger}$ There exist a continuous function $B: I R \times I R^{n} \rightarrow I R^{n, m}$, a candidate Lyapunov function $V: I R \times I R^{n} \rightarrow I R_{+}$, a class $K$ function $\gamma: I R_{+} \rightarrow I R_{+}$, functions $\beta_{1}, \beta_{2}: I R \times I R^{n} \times \Omega \rightarrow I R_{+}$and continuous functions $\kappa, \rho: I R \times I R^{n} \rightarrow I R_{+}$ such that

$F(t, x, u, \omega)=f_{s}(t, x, \omega)+B(t, x) g(t, x, u, \omega)$

$\dagger^{\dagger}$ For definition see (Corless et al., 1988; Corless et al., 1990.) 
for some functions $f_{s}$ and $g$ which satisfy:

1) For each $\omega \in \Omega, f_{s}(\cdot, \omega)$ is continuous and

$\frac{\partial V}{\partial t}(t, x)+\frac{\partial V}{\partial x}(t, x) f_{s}(t, x, \omega) \leq-\gamma(\|x\|)$

for all $t \in I R, x \in I R^{n}$.

2) For each $\omega \in \Omega, g(\cdot \omega)$ is continuous and

$u^{T} g(t, x, u, \omega) \geq-\beta_{1}(t, x, \omega)\|u\|+\beta_{2}(t, x, \omega)\|u\|^{2}$

where

$\beta_{1}(t, x, \omega) \leq \beta_{2}(t, x, \omega) \rho(t, x)$

$\beta_{1}(t, x, \omega) \leq \kappa(t, x)$

for all $t \in I R, x \in I R^{n}, u \in I R^{m}$.

\subsection{Proposed Controllers}

Here we present some practically stabilizing controller sets for the system considered in the previous section. These controllers can be regarded as continuous approximations of those presented in (Gutman and Leitmann, 1976).

Consider any uncertain system described above and let $(B, V, \gamma, \rho, \kappa)$ be a quintuple which assures the satisfaction of assumption C.1. Choose any continuous functions $\rho_{c}, \kappa_{c}$ which satisfy

$\rho_{c}(t, x) \geq \rho(t, x), \kappa_{c}(t, x) \geq \kappa(t, x)$

and define

$\alpha(t, x):=B(t, x)^{T} \frac{\partial V}{\partial x}(t, x)^{T}$,

$\eta(t, x):=\kappa_{c}(t, x) \alpha(t, x)$.

A practically stabilizing family of controllers is the set

$\mathrm{P}:=\left\{p_{\epsilon} \mid \epsilon>0\right\}$

where $p_{\epsilon}$ is any continuous function which satisfies

$\|\alpha(t, x)\| p_{\varepsilon}(t, x)=-\left\|p_{\varepsilon}(t, x)\right\| \alpha(t, x)$

i.e., $p_{\epsilon}(t, x)$ is opposite in direction to $\alpha(t, x)$, and

$\|\eta(t, x)\| \neq 0 \Longrightarrow\left\|p_{c}(t, x)\right\| \geq \rho_{c}(t, x)\left[1-\|\eta(t, x)\|^{-1} \epsilon\right]$. 
As an example of a function satisfying the above requirements on $p_{\epsilon}$, consider

$p_{\epsilon}(t, x):= \begin{cases}-\frac{\eta(t, x)}{\epsilon} \rho_{c}(t, x) & \text { if }\|\eta(t, x)\| \leq \epsilon \\ -\frac{\eta(t, x)}{\|\eta(t, x)\|} \rho_{c}(t, x) & \text { if }\|\eta(t, x)\|>\epsilon ;\end{cases}$

see (Corless and Leitmann, 1981).

As another example, consider

$p_{c}(t, x):=-\frac{\eta(t, x)}{\|\eta(t, x)\|+\epsilon} \rho_{c}(t, x)$

see (Ambrosino et al., 1985).

Controllers of a discontinuous type as well as their continuous approximations, related to those proposed here, have been deduced by employing the theory of variable structure control; see (Bartolini and Zolezzi, 1985). Some early treatments of controller design for uncertain systems were based on "games against nature"; see (Gutman and Leitmann, 1975). Another class of controllers for systems of type (1) are deduced in (Barmish et al., 1983).

\subsection{Matching Conditions}

Given a system described by (1) the choice of $B, f_{s}, g$ to assure satisfaction of Assumption $C .1$ (if possible) may not be obvious. This choice is usually easier if the uncertainties are matched in the sense that there exist functions $f_{0}, B, g$ with $B(t, x) \in I R^{n, m}$ such that

$F(t, x, u, \omega)=f_{0}(t, x)+B(t, x) g(t, x, u, \omega) ;$

that is, the uncertainty $\omega$ and the control enter the system description via the same matrix $B(t, x)$.

Much of the literature concerns systems in which the uncertainties are matched. (Barmish and Leitmann, 1982) and (Chen and Leitmann, 1987) consider systems with unmatched uncertainties; there the norm of the unmatched portion of the uncertain term must be smaller than a certain threshold value. In (Stalford, 1987a) linear systems are considered in which the uncertainty satisfies generalized matching conditions, that is, structural conditions which are less restrictive than the matching condition. In these cases, as in the matched case, the norm bounds of the uncertain terms can be arbitrarily large. Linear time-invariant systems with scalar control input are treated in (Stalford, $1987 \mathrm{~b}$ ), while (Schmitendorf, 1988) requires the existence of a positive definite solution of a certain Riccati equation.

\subsection{Other Problems}

While global uniform asymptotic stability or at least practical stability can be guaranteed provided the control is not constrained, only local stability can be assured if the available control is subject to constraints. One class of stabilization problems with control constraints is considered by (Soldatos et al., 1991, Corless and Leitmann, 1993). Controllers 
which assure not only practical stability but also exponential convergence at a prescribed rate are treated in (Corless et al., 1988, Corless, 1993). (Corless and Leitmann, 1983) deal with systems in which the uncertainty bounds are not known exactly but depend on unknown constants; the controllers presented there are parameter adaptive controllers. Problems in which one wishes to keep the system state within or outside a prescribed region of the state space are considered in (Corless et al., 1987). Systems with delay are considered in (Thowsen, 1983) and (Lee and Leitmann, 1988). (Ha and Gilbert, 1987) treat controllers which linearize a nominal system in addition to assuring stability of the actual one. Large scale uncertain systems with decentralized control are discussed in (Chen, 1987a) and (Siljak, 1991).

\section{DISCRETE SYSTEMS CONTROL}

The control of uncertain discrete systems modelled by difference equations of the form (2) has been treated in (Corless and Manela, 1986, Magana and Zak, 1988 and Sezer and Siljak, 1988). Unlike in the continuous case reviewed in the previous section, arbitrarily large uncertainties cannot be tolerated, in general, and the region of ultimate attraction cannot be made arbitrarily small. (Corless and Manela, 1986) consider the matched case, namely

$x(k+1)=f(k, x(k))+B(k, x(k))[u(k)+e(k, x(k), u(k))]$

where $k \in \mathbb{Z}, x(k) \in I R^{n}$ and $u(k) \in I R^{m}$. The functions $f: \mathbb{Z} \times I R^{n} \rightarrow I R^{n}$ and $B: \mathbb{Z} \times I R^{n} \rightarrow I R^{n, m}$ are assumed known, with

$\operatorname{rank}[B(k, x(k))]=m$.

The function $e: \mathbb{Z} \times I R^{n} \times I R^{m} \rightarrow I R^{m}$ is not known; however, it is assumed that the class of functions $E$ to which it belongs is known. We make the following two assumptions before stating a stabilization theorem.

Assumption D.1. $\ddagger$ Given a positive definite $P \in I R^{n, n}$ there exist non-negative scalars $\rho_{0}, \rho_{1}$ and $\rho_{2}$ such that for all $e \in \mathrm{E}$

$\|B(k, x) e(k, x, u)\|_{P} \leq \rho_{0}+\rho_{1}\|x\|_{P}+\rho_{2}\|u\|_{R(k, x)}$

for all $(k, x, u) \in \mathbb{Z} \times I^{n} \times I R^{m}$, where $R(k, x):=B(k, x)^{T} P B(k, x)$. Next we define

$$
\begin{aligned}
\psi(k, x) & :=\left[B(k, x)^{T} P B(k, x)\right]^{-1} B(k, x)^{T} P, \\
\phi(k, x) & :=B(k, x) \psi(k, x), \\
\bar{f}(k, x) & :=\phi(k, x) f(k, x),
\end{aligned}
$$

Let $P \in I R^{n, n}$ be a positive definite matrix. We define the norm

$\|\cdot\| P: I R^{n} \rightarrow I R_{+}$by $\|r\|_{P}:=\sqrt{r^{T} P r}$. 
and

$\tilde{f}(k, x):=f(k, x)-\bar{f}(k, x)$.

Assumption D.2. There exist a positive definite matrix $P \in I R^{n, n}$ and a non-negative scalar $\tilde{c}<1$ such that

$\|\tilde{f}(k, x)\|_{P} \leq \tilde{c}\|x\|_{P}$

for all $(k, x) \in \mathbb{Z} \times I R^{n}$. If $\rho_{2} \neq 0$, then there also exist non-negative scalars $c_{0}$ and $c_{1}$ such that

$\|\vec{f}(k, x)\|_{P} \leq c_{0}+c_{1}\|x\|_{P}$

for all $(k, x) \in \mathbb{Z} \times I R^{n}$.

\subsection{Proposed Controllers}

Consider an uncertain discrete system (20) satisfying assumptions D.1. - D.2 and subject to the control $u(k)=p(k, x(k))$ where $p(k, x(k))$ is defined as follows:

$p(k, x(k)):= \begin{cases}0 & \text { if } \rho_{2} \geq 1 \\ -\Psi(k, x(k)) f(k, x(k)) & \text { if } \rho_{2}<1 .\end{cases}$

Suppose that

$\tilde{c}^{2}+\left(\rho_{1}+c_{1}^{*}\right)^{2}<1$

where

$c_{1}^{*}:= \begin{cases}0 & \text { if } \rho_{2}=0 \\ \rho_{2}^{*} c_{1} & \text { if } \rho_{2} \neq 0\end{cases}$

and

$\rho_{2}^{*}:=\min \left\{\rho_{2}, 1\right\}$.

Then for all $e \in \mathrm{E}$, the feedback controlled system (20) is g.u.a.s. about the set

$B_{P}(d):=\left\{x \in I R^{n} \mid\|x\|_{P} \leq d\right\}$

where

$d:=\frac{\rho_{0}+c_{0}^{*}}{\sqrt{1-\tilde{c}^{2}-\left(\rho_{1}+c_{1}^{*}\right)}}$ 
and

$c_{0}^{*}:= \begin{cases}0 & \text { if } \rho_{2}=0 \\ \rho_{2}^{*} c_{0} & \text { if } \rho_{2} \neq 0\end{cases}$

\section{ROBUSTNESS IN THE PRESENCE OF SINGULAR PERTURBATIONS}

Consider an uncertain singularly perturbed system described by

$$
\begin{aligned}
& \dot{x}=F(t, x, y, u, \mu, \omega) \\
& \mu \dot{y}=G(t, x, y, u, \mu, \omega)
\end{aligned}
$$

where $(x, y) \in I R^{n} \times I R^{l}$ describe the state of the system. $\mu \in(0, \infty)$ is the singular perturbation parameter, and all the other variables are as described above. Here one wants to obtain memoryless feedback controllers (generating $u$ ) which assure that, for all $\omega \in \Omega$ and for all sufficiently small $\mu$, the behavior of the feedback controlled system is close to that of g.u.a.s.

Assuming that, for each $x, u, \omega$ there exists a unique vector $H(x, u, \omega) \in I R^{l}$ such that

$G(t, x, H(x, u, \omega), u, 0, \omega)=0$

for all $t$, the reduced order system associated with (36) (let $\mu=0$ in (36)) is given by

$\dot{x}=\bar{F}(t, x, u, \omega)$

where

$\bar{F}(t, x, u, \omega):=F(t, x, H(x, u, \omega), u, 0, \omega)$.

For each $t, x, u, \omega$ the boundary layer system associated with (36) is given by

$\frac{d y}{d \tau}(\tau)=G(t, x, y(\tau), u, 0, \omega)$

(Corless et al., 1990) require that the boundary layer system satisfies g.u.a.s. about its equilibrium state $H(x, u, \omega)$ and present stabilizing controllers whose designs are based on the reduced order system. This situation occurs for systems with stable "neglected dynamics." In (Garofalo and Leitmann, 1990) the boundary layer system is not required to be stable. The "stabilizing" controllers presented there are composite controllers in the sense that they consist of two parts; one part is utilized to stabilize the boundary layer system and the other part is based on a nominal reduced order system. 


\section{OUTPUT FEEDBACK}

Heretofore it was assumed that the complete state is available for feedback. Consider now the more general situation in which the output $y(t) \in I R^{s}$ available for feedback is related to the state by

$y(t)=c(t, x(t), \omega)$

for some function $c: I R \times I R^{n} \times \Omega \rightarrow I R^{s}$.

Memoryless output feedback controllers are treated in (Galimidi and Barmish, 1986, Steinberg and Ryan, 1986 and Chen 1987b). The dynamic output feedback controllers in the literature utilize state estimators. The state is fed to a memoryless controller whose design is based on having the complete state available for feedback. Full order observers are utilized in (Barmish and Galimidi, 1986 and Walcott and Zak, 1987). (Breinl and Leitmann, 1983 and Breinl and Leitmann, 1987) utilize reduced order observers. There the uncertain terms must satisfy certain structural conditions and the differential equation describing the evolution of the state estimation error is decoupled from the state equation.

\section{APPLICATIONS}

Controller designs based on a constructive use of Lyapunov stability theory or closely related methods have been applied to a variety of uncertain systems. In the realm of engineering these applications include tracking control for robotic manipulators including hybrid tracking and force control (Reithmeier and Leitmann, 1991), suspension control for magnetically levitated vehicles (Breinl and Leitmann, 1983 and Breinl and Leitmann, 1987), control of seismically excited structures (Kelly et al., 1987), of high speed rotors (Weltin, 1988), and of nuclear power plants (Parlos et al., 1988), as well as various aircraft and aerospace systems (Singh, 1987, Stalford, 1987b, Leitmann and Pandey, 1991). Experimental results may be found in (Horowitz et al., 1989 and Kang et al., 1991). (Deissenberg, 1986 and Leitmann and Wan, 1978) concern applications in economics. Resource allocation in fisheries is discussed in (Kaitala and Leitmann, 1990, Kaitala and Leitmann, 1992, Hilden et al., 1993). Harvesting problems are treated in (Lee and Leitmann, 1983 and Corless and Leitmann, 1985). (Lee and Leitmann, 1987 and Lee and Leitmann, 1988) deal with pollution control in rivers. (Lee and Leitmann, 1991) treat a problem in pedagogy. In the complete version of the paper presented here, two examples of resource management based on uncertain models, one continous and the other discrete, are discussed; see (Leitmann, 1995).

\section{REFERENCES}

Ambrosino, G., Celentano, G. and Garofalo, F. (1985) Robust Model Tracking Control for a Class of Nonlinear Plants. IEEE Trans. Automatic Control, AC-30, 275.

Barmish, B.R., Corless, M., Leitmann, G. (1983) A new class of stabilizing controllers for uncertain dynamical systems. SIAM J. on Control and Optimization, 21, No. 2, 246. 
Barmish, B.R., Galimidi, A.R. (1986) Robustness of Luenberger Observers: Linear System Stabilized via Nonlinear Control. Automatica 22, 246.

Barmish, B.R., Leitmann, G. (1982) On Ultimate Boundedness Control of Uncertain Systems in Absence of Matching Conditions. IEEE Trans. Automatic Control, AC-27, 1253.

Bartolini G., Zolezzi, T. (1985) Variable Structure Systems Nonlinear in the Control Law. IEEE Trans. Automatic Control, AC-30, 681.

Bass, R.W. (1985) Discussion of: "Die Stabilität von Regelsystemen mit nachgebender Rückführung" by A.M. Letov. in Proc. Heidelberg Conf. Automatic Control.

Breinl, W., Leitmann, G. (1983) Zustandsrückführung für dynamische Systeme mit Parameterunsicherheiten. Zeitschrift für Regelungstechnik, 31, 95.

Breinl, W., Leitmann, G. (1987) State Feedback for Uncertain Dynamical Systems. Applied Mathematics and Computation, 22, 65.

Chen, Y.H. (1987a) Deterministic Control of Large Scale Uncertain Dynamical Systems. J. Franklin Inst., 323, 125.

Chen, Y.H. (1987b) Robust Output Feedback Controller: Direct Design. Int. J. Control, 46, 1083.

Chen, Y.H., Leitmann, G. (1987) Robustness of Uncertain Systems in the Absence of Matching Assumptions. Int. Journal of Control, 45, 1527.

Corless, M. (1993) Control of Uncertain Nonlinear Systems. ASME J. of Dynamical Systems, Measurement, and Control, 115, 362.

Corless, M., Garofalo, F., Leitmann, G. (1988) Guaranteeing Exponential Convergence for Uncertain Systems, Proc. Int. Workshop on Robustness in Identification and Control, Torino, Italy.

Corless, M., Leitmann, G. (1981) Continuous State Feedback Guaranteeing Uniform Ultimate Boundedness for Uncertain Dynamic Systems. IEEE Trans. Automatic Control, AC-26, 1139.

Corless, M., Leitmann G. (1983) Adaptive Control of Systems Containing Uncertain Functions and Unknown Functions with Uncertain Bounds. J. Optimiz. Theory Applic., 41, 155.

Corless, M., Leitmann, G. (1985) Adaptive Long-Term Management of Some Ecological Systems Subject to Uncertain Disturbances, in Optimal Control Theory and Economic Analysis 3 (ed. G. Feichtinger), Elsvier Science Publishers, Amsterdam, Holland.

Corless, M., Leitmann, G. (1988) Deterministic Control of Uncertain Systems, in Proc. Conf. on Modeling and Adaptive Control, Sopron, Hungary, Lecture Notes in Control and Information Sciences, Springer Verlag, IIASA 105.

Corless, M., Leitmann, G. (1990) Deterministic Control of Uncertain Systems: A Lyapunov Theory Approach, in Deterministic Control of Uncertain Systems, Chapter 11, (ed. A.S.I. Zinober), Peter Peregrinus, London.

Corless, M., Leitmann, G. (1993) Bounded Controllers for Robust Exponential Convergence. Optimiz. Theory Appl., 76, 1.

Corless, M., Leitmann, G., Ryan, E.P. (1990) Uncertain Systems With Neglected Dynamics, in Deterministic Control of Uncertain Systems, Chapter 12, (ed. A.S.I. Zinober), Peter Perigrinus, London.

Corless, M., Leitmann, G., Skowronski, J.M. (1987) Adaptive Control for Avoidance or Evasion in an Uncertain Environment. Computers and Mathematics with Applicaitons, 13, 1 . 
Corless, M., Manela, J. (1986) Control of Uncertain Discrete-Time Systems, in Proc. American Control. Conf., Seattle, Washington.

Galimidi, A.R., Barmish, B.R. (1986) The Constrained Lyapunov Problem and its Application to Robust Output Feedback Stabilization, IEEE Trans. Automatic Control, AC-31, 410.

Garofalo, F., Leitmann, G. (1990) Nonlinear Composite Control of a Clașs of Nominally Linear Singularly Perturbed Uncertain Systems, in Deterministic Control of Uncertain Systems, Chapter 13, (ed. A.S.I. Zinober), Peter Peregrinus, London.

Gutman, S., Leitmann, G. (1975) On a Class of Linear Differential Games. J. Optimiz. Theory Appl., 17, 511.

Gutman, S., Leitmann, G. (1976) Stabilizing Feedback Control for Dynamical Systems with Bounded Uncertainty, in Proc. IEEE Conf. Decision Control, Clearwater, Florida.

Ha, I.-J., Gilbert, E.G. (1987) Robust Tracking in Nonlinear Systems. IEEE Trans. Automatic Control, AC-32, 763.

Hilden, M., Kaitala, V., Leitmann, G. (1994) Stabilizing Management and Structural Development of Open Access Fisheries, in Advances in Dynamic Games and Applications, (eds. T. Basar and A. Haurie), Birkhäuser Verlag, Basel, Switzerland.

Horowitz, R., Stephens, H.I., Leitmann, G. (1989) Experimental Implementation of a Deterministic Controller for a D.C. Motor with Uncertain Dynamics. J. Dynam. Syst. Meas. Control, 111, 244.

Kaitala, V., Leitmann, G. (1990) Stabilizing Employment in a Fluctuating Recource Economy, J. Optimiz. Theory Appl., 67, 1.

Kaitala, V., Leitmann, G. (1992) Income Subsidizing and Fisheries Development - An Analysis of Stabilizing Management, in Dynamic Economic Models and Optimal Control, (ed. G. Feichtinger), Elsevier Science Publishers, Amsterdam, Holland.

Kang, C.G., Horowitz, R., Leitmann, G. (1991) Robust Deterministic Control for Robotic Manipulators, in Proceed. ASME, Annual Meeting.

Klir, G.J., Folger, T.A. (1988) Fuzzy Sets, Uncertainty and Information. Prentice Hall, Englewood Cliffs, N.J.

Kurzhanskii, A.B. (1983) Evolution Equations for Problems of Control and Estimation of Uncertain Systems. Proc. Int. Cong. Math., Warsaw, Poland.

Lee, C.S., Leitmann, G. (1983) On Optimal Long-Term Management of Some Ecological Systems Subject to Uncertain Disturbances. Int. J. Syst. Sci, 14, 979.

Lee, C.S. Leitmann, G. (1987) Uncertain Dynamical Systems: An Application to River Polution Control, in Proc. Modeling and Management of Resources Under Uncertainty, Honolulu, Lecture Notes in Biomathematics, 72, Springer Verlag, Berlin.

Lee, C.S., Leitmann, G. (1988) Continuous Feedback Guaranteeing Uniform Ultimate Boundedness for Uncertain Linear Delay Systems: An Application to River Pollution Control. Comput. Math. Appl., 16, 929.

Lee, C.S., Leitmann, G. (1991) Some stabilizing study strategies for a student-related problem under uncertainty. Dynamics and Stability of Systems, 6, No. 1, 63.

Leitmann, G. (1990) Deterministic Control of Uncertain Systems Via a Constructive Use of Lyapunov Stability Theory, in Proceed. 14th IFIP Conf., Leipzig, 1989, Lecture Notes in Control and Information Sciences, 143, Springer Verlag, Berlin.

Leitmann, G. (1993) On One Approach to the Control of Uncertain Systems. ASME J. of Dynamical Systems, Measurement, and Control, 115, 373. 
Leitmann, G. (1995) One Method for Robust Control of Uncertain Systems - Theory and Practice. Kybernetika, to appear.

Leitmann, G., Pandey, S. (1991) Aircraft Control for Flight in an Uncertain Environment: Takeoff in Windshear. J. Optimiz. Theory Appl., 70, (1).

Leitmann, G., Wan, H.Y. Jr. (1978) A Stabilization Policy for an Economy with Some Unknown Characteristics. J. Franklin Inst., 306, 23.

Magana, M.E., Zak, S.H. (1988) Robust Output Feedback Stabilization of Discrete-Time Uncertain Dynamical Systems, IEEE Trans. Automatic Control, AC-33, 1082.

Parlos, A.G., Henry, A.F. Schweppe, F.C., Gould, L.A., Lanning, D.D. (1988) Nonlinear Multivariable Control of Nuclear Power Plants Based on the Unknown-but-bounded Disturbance Model. IEEE Trans. Automatic Control, AC-33, 130.

Reithmeier, E., Leitmann, G. (1991) Tracking and Force Control for a Class of Robotic Manipulators. Dynamics and Control, 1(2).

Schmitendorf, W.E. (1988) Stabilizing Controllers for Uncertain Linear Systems with Additive Disturbances. Int. J. Control47, 85.

Sezer, M.E., Siljak, D.D. (1988) Robust Stability of Discrete Systems. Int. J. Control, 48, 2055.

Siljak, D.D. (1991) Decentralized Control of Complex Systems. Academic Press, New York.

Singh, S.N. (1987) Attitude Control of a Three Rotor Gyrostat in the Presence of Uncertainty. J. Astronautical Sciences, $\mathbf{3 5}$.

Soldatos, A.G., Corless, M., Leitmann, G. (1991) Stabilizing Uncertain Systems with Bounded Control, in Third Workshop on Control Mechanics, Lecture Notes in Control and Information Sciences, 409, Springer Verlag, Berlin.

Stalford, H.L. (1987a) Robust Control of Uncertain Systems in the Absence of Matching Conditions: Scalar Input, in Proc. IEEE Conf. Decision and Control.

Stalford, H.L. (1987b) On Robust Control of Wing Rock Using Nonlinear Control, in Proc. American Control Conf., Minneapolis, Minn.

Steinberg, A., Ryan, E.P. (1986) Dynamic Output Feedback Control of a Class of Uncertain Systems. IEEE Trans. Control, AC-31, 1163.

Thowsen, A. (1983) Uniform Ultimate Boundedness of the Solutions of Uncertain Dynamic Delay Systems with State-Dependent and Memoryless Feedback. Int. J. Control, 37, 1135.

Walcott, B.L., Zak, S.H. (1987) State Observation of Nonlinear Uncertain Dynamical Systems. IEEE Trans.Automatic Control, AC-32, 166.

Weltin, U. (1988) Aktive Schwingungsdämpfung von Rotoren mit Parameterunsicherheiten. Dr. - Ing. Dissertation, TU Darmstadt, Germany. 